ARTICLE

\title{
Structural basis for assembly of non-canonical small subunits into type I-C Cascade
}

Roisin E. O'Brien', Inês C. Santos ${ }^{2}$, Daniel Wrapp (1 ${ }^{3}$, Jack P. K. Bravo ${ }^{3}$, Evan A. Schwartz ${ }^{1}$, Jennifer S. Brodbelt ${ }^{2}$ \& David W. Taylor (10) 1,3,4,5凶

Bacteria and archaea employ CRISPR (clustered, regularly, interspaced, short palindromic repeats)-Cas (CRISPR-associated) systems as a type of adaptive immunity to target and degrade foreign nucleic acids. While a myriad of CRISPR-Cas systems have been identified to date, type I-C is one of the most commonly found subtypes in nature. Interestingly, the type I$C$ system employs a minimal Cascade effector complex, which encodes only three unique subunits in its operon. Here, we present a $3.1 \AA$ resolution cryo-EM structure of the Desulfovibrio vulgaris type I-C Cascade, revealing the molecular mechanisms that underlie RNAdirected complex assembly. We demonstrate how this minimal Cascade utilizes previously overlooked, non-canonical small subunits to stabilize R-loop formation. Furthermore, we describe putative PAM and Cas3 binding sites. These findings provide the structural basis for harnessing the type I-C Cascade as a genome-engineering tool.

\footnotetext{
${ }^{1}$ Institute for Cell and Molecular Biology, University of Texas at Austin, Austin, TX 78712, USA. ${ }^{2}$ Department of Chemistry, University of Texas at Austin Austin, TX 78712, USA. ${ }^{3}$ Department of Molecular Biosciences, University of Texas at Austin, Austin, TX 78712, USA. ${ }^{4}$ Center for Systems and Synthetic Biology, University of Texas at Austin, Austin, TX 78712, USA. ${ }^{5}$ Livestrong Cancer Institutes, Dell Medical School, Austin, TX 78712, USA.

凶email: dtaylor@utexas.edu
} 
C RISPR-RNA (clustered, regularly, interspaced, short palindromic repeats-RNA) along with Cas proteins assemble into RNA-guided adaptive immune complexes in prokaryotes ${ }^{1}$. These CRISPR-Cas systems defend bacteria and archaea against the invasion of foreign genetic elements ${ }^{2}$. CRISPR-Cas systems can be divided into two major classes based on their targeting complexes: multi-subunit effector (Class I) or a single protein effector (Class II) ${ }^{3}$. The type I-C subtype is one of the most prevalent systems found in bacteria ${ }^{4}$. However, relatively little information exists about its effector complex.

Interestingly, type I-C Cascade only contains three unique Cas proteins in its operon: Cas $5 c$, Cas7, and Cas $8 c^{3}$ (Fig. 1a). The type I-C Cascade uses Cas5c for processing the crRNA instead of a separate Cas6 (refs. ${ }^{3,5,6}$ ) and does not include a small subunit (SSU) within its operon ${ }^{3}$, making this a minimal Cascade (Fig. 1a). Previous studies hypothesized that the large subunit, Cas8c, was a fusion of the larger and smaller subunits found in the type I-E Cascade $^{6}$. However, a recent report revealed that the Desulfovibrio vulgaris Cas8c large subunit includes an internal ribosome-binding site at the $\mathrm{C}$ terminus, which encodes a separate $\mathrm{SSU}^{7}$. This noncanonical SSU was shown to be equivalent to the Cas11 SSU found in type I-E and appeared widespread within the I-B, I-C, and I-D subtypes ${ }^{7}$. Here, we demonstrate that this non-canonical subunit is an integral component within the complex and is primed for stabilizing the non-target strand during R-loop formation.

\section{Results}

Stoichometry, assembly, and cryo-electron microscopy (cryoEM) structure of type I-C Cascade complex. We purified the $D$. vulgaris type I-C Cascade from Escherichia coli, which revealed the presence of an additional $14 \mathrm{kDa}$ protein, corresponding to the recently identified SSU (Supplementary Fig. 1). We then analyzed the complex using native mass spectrometry $(\mathrm{MS})^{8-11}$, which exhibited the presence of two dominant species with masses of 275 and $371 \mathrm{kDa}$, respectively (Fig. 1b). The larger species $(371 \mathrm{kDa})$ corresponds to a fully intact type I-C Cascade with a stoichiometry of Cas $7_{7} \mathrm{Cas} 8 \mathrm{c}_{1} \mathrm{Cas} 5 \mathrm{c}_{1} \mathrm{SSU}_{2} / \mathrm{crRNA}_{1}$. The smaller species $(275 \mathrm{kDa})$ is consistent with the Cascade lacking Cas5c and Cas $8 \mathrm{c}$ or lacking the two SSUs and Cas8c. Since previous isothermal titration calorimetry experiments ${ }^{6}$ have demonstrated that Cas $5 \mathrm{c}$ has a higher affinity for the crRNA than Cas $8 \mathrm{c}$; the $275 \mathrm{kDa}$ subcomplex most likely represents Cascade after dissociation of the SSUs and Cas8c due to weakening of hydrophobic interactions within the gas phase ${ }^{12}$. Application of gentle collisional activation via in-source trapping (IST) was used to disassemble the complexes prior to mass analysis, thus allowing inspection of the composition of the individual subunits and the architecture of subcomplexes (Fig. 1b, insets). The theoretical and experimental masses obtained from native mass spectra with IST are provided in Supplementary Table 1.

To understand the molecular basis for small-subunit incorporation, we determined a $3.1 \AA$ resolution cryo-EM reconstruction of the type I-C Cascade complex (Fig. 1c, Supplementary Figs. 2-4, and Supplementary Table 2), suitable for de novo model building (except for the flexible $\mathrm{N}$ terminus of Cas8c) (Supplementary Fig. 5). The overall architecture of the complex resembles a caterpillar. Seven Cas7 subunits form a right-handed helical filament around the crRNA and Cas5c sits at the base of the complex (Fig. 1d). Cas5c and Cas7.7 clamp around the crRNA $5^{\prime}$-handle (nucleotides U1-G12), forcing it into a hooked conformation (Fig. 1d, inset). Cas $5 c$ residues "pinch" the phosphate groups within the crRNA backbone on either side of

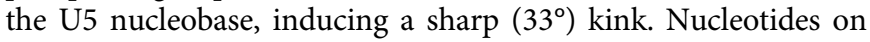
either side of this kink are captured by a network of Cas $5 c \pi-\pi$ stacking interactions, while Cas7.7 makes non-specific contacts with the phosphate backbone (Supplementary Fig. 6). These highly conserved interactions (Fig. 1d, inset) suggest that the $5^{\prime}$ end of the crRNA handle is critical for type I-C Cascade assembly.

Seven Cas7 subunits span the length of the crRNA and are capped by the $3^{\prime}$ end (Fig. 1c). While type I-E and type I-F Cascades incorporate a Cas6 subunit, an additional Cas7 subunit forms the head of the type I-C Cascade ${ }^{13,14}$ (Fig. 1c). Interestingly, when the bottom Cas7 subunits from type I-F, I-E, IIII-A, and III-B are all aligned to the type I-C Cas7.1, the type I-C crRNA backbone more closely resembles that of type III-A and -B complexes (root-meansquare deviation (RMSD) $7.8 \AA$ ), rather than the type I-E (RMSD $10.6 \AA$ ) or type I-F (RMSD 19.1 $\AA$ ) Cascades (Fig. 1e) ${ }^{8-11}$. The type III-A, type III-B, and type I-C crRNA lack a $3^{\prime}$ stem-loop, which correlates with a more linear geometry of the crRNA backbone ${ }^{15,16}$ (Fig. 1e). Despite these differences, type I-C Cas7 maintains a highly conserved region of positive residues to form non-specific interactions with the phosphate backbone of the crRNA. (Fig. 1e, inset, and Supplementary Fig. 7).

The belly of the complex contains the large subunit, Cas8c, and two copies of the SSU, which nucleate and are derived from the Cterminal domain of Cas8c (residues 489-612) (Fig. 1c). These SSUs are structurally identical to the C-terminal domain of Cas8c (RMSD of 0.59 and $0.67 \AA$ for SSU.1c and SSU.2c to Cas $8 \mathrm{c} C$ terminus, respectively) (Fig. 2a) and adopt a helical bundle topology typical of other SSUs ${ }^{8-11}$ (Fig. 2b). In the type I-E system, the Cse2 SSUs are responsible for supporting the non-target strand during R-loop formation (Supplementary Fig. 8). Remarkably, the electrostatic surface potential of the type I-C Cascade (Fig. 2c) reveals a contiguous channel of positively charged residues that runs along the length of this minor filament from the large subunit (Fig. 2c). We then compared our model with a previous lower-resolution reconstruction of type I-C Cascade ${ }^{6}$ (Fig. 2d). As anticipated, additional density corresponding to the non-target strand follows the positively charged path across the surface of the SSU (Fig. 2d, inset), indicating that these non-canonical SSUs may accommodate the non-target strand during DNA targeting.

Structural insights into PAM recognition and Cas $3 c$ recruitment. In the type I-E Cascade, the large subunit Cse1 is responsible for identifying the PAM (protospacer adjacent motif) site on the non-target strand of the dsDNA target $17-19$. Notably, the overlay of the target DNA density shows Cas8c is in a position to interact with the PAM sequence in the duplex. A glycine loop and adjacent positively charged residues create a putative PAM binding site (Fig. 2d, inset) located near position 1- and 0-nt (C11 and G12), which are required for target recognition. Following PAM recognition, a trans-acting nuclease-helicase Cas3 subunit is recruited for target degradation in most type I systems, and interacts exclusively with the large subunit ${ }^{20-22}$. To understand Cas $3 \mathrm{c}$ recruitment, we generated a homology model of Cas $3 \mathrm{c}$ and predicted its Cas8c-interacting surfaces using MorphProt ${ }^{23}$, revealing regions of complementary charges and hydrophobicity located on the surface of Cas8c and Cas3c (Fig. 2e). This binding site positions Cas $3 \mathrm{c}$ to favorably interact with the non-target strand during R-loop formation (Fig. 2f) and is consistent with previously reported Cas3-bound Cascade structures ${ }^{21}$.

\section{Discussion}

Our structural work provides the first molecular insights into the sequence-specificity of Cas $5 \mathrm{c}-\mathrm{crRNA}$ interactions and nonspecific Cas7-crRNA interactions that are critical for type I-C Cascade assembly. The Cas5c-Cas7.7 clamp around the crRNA nucleates Cascade complex assembly, which is likely followed by cooperative assembly of the Cas7 backbone. This culminates in the addition of the Cas8c-Cas11.1c-Cas11.2c "belly" architecture. 
a
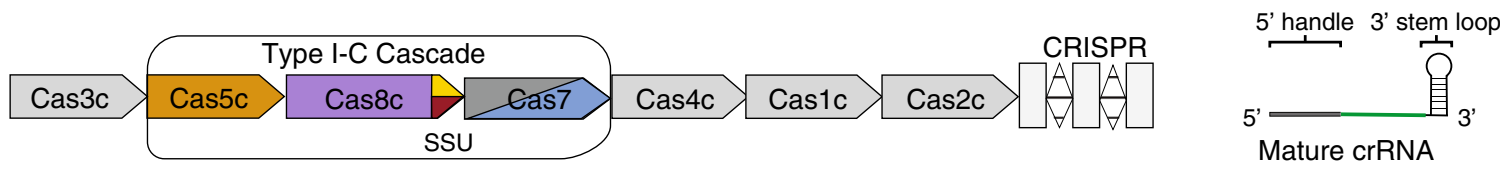

(64-68 nt)
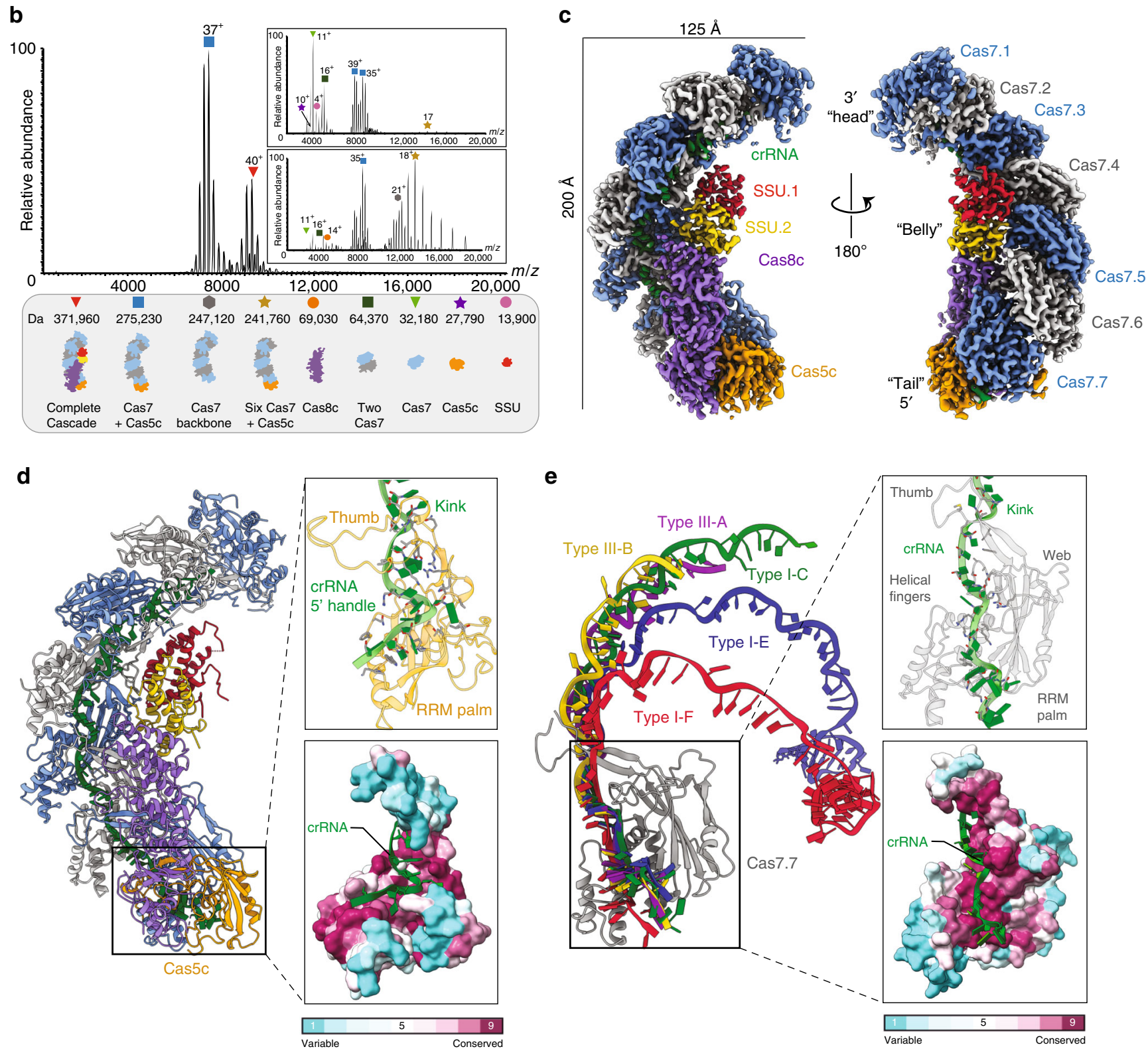

Fig. 1 Architecture of type I-C Cascade. a The D. vulgaris type I-C operon (left) and mature crRNA (right). b ESI mass spectrum of the type I-C Cascade confirms the presence of a non-canonical SSU. Gentle disassembly of the complexes prior to mass analysis using two different desolvation voltages ( -120 and $-300 \mathrm{~V}$ ) allows the composition of the individual subunits and the architecture of the complexes to be determined (inset top and bottom). All complexes/subcomplexes detected and associated experimental masses are annotated. c Cryo-EM structure of the type I-C Cascade at 3.1- $\AA$ resolution. The structure is segmented and colored as follows: Cas7, blue and gray; Cas8c, purple; Cas5, orange; Cas11c, gold and red; crRNA, green. $\mathbf{d}$ The resulting atomic model colored as in c. Cas $5 \mathrm{c}$ (orange) resembles a right-handed fist with an RRM palm and thumb domains. Residues (gray) interacting with the $5^{\prime}$ handle (green) (inset top) are highly conserved (inset bottom). e Alignment of type III-B (yellow), type I-E (blue), type I-F (red), type III-A (purple) Cas7 tail to type I-C Cas7.7 demonstrates variability in crRNA backbone orientation. Residues (dark gray) interacting with the crRNA (green) (inset top) are highly conserved (inset bottom).

This hierarchical assembly is supported by our native MS data, which demonstrate that Cas5c-Cas7-crRNA form a stable complex in the absence of Cas8c and Cas11c (Fig. 1b). We reveal how the incorporation of a previously overlooked SSU may stabilize the non-target strand during R-loop formation. Furthermore, we identify distinct, exposed surfaces on Cas8c that creates a central hub for DNA duplex separation, PAM recognition, Cas $3 c$ recruitment, and ultimately dsDNA degradation by the minimal type I-C Cascade (Fig. 2f). Taken together, our model provides functional insights into one of the most prevalent CRISPR-Cas 
a

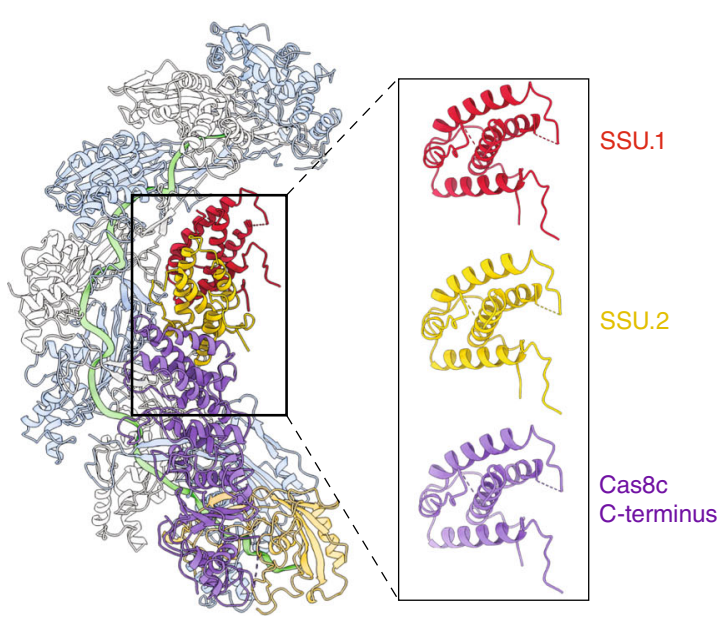

C
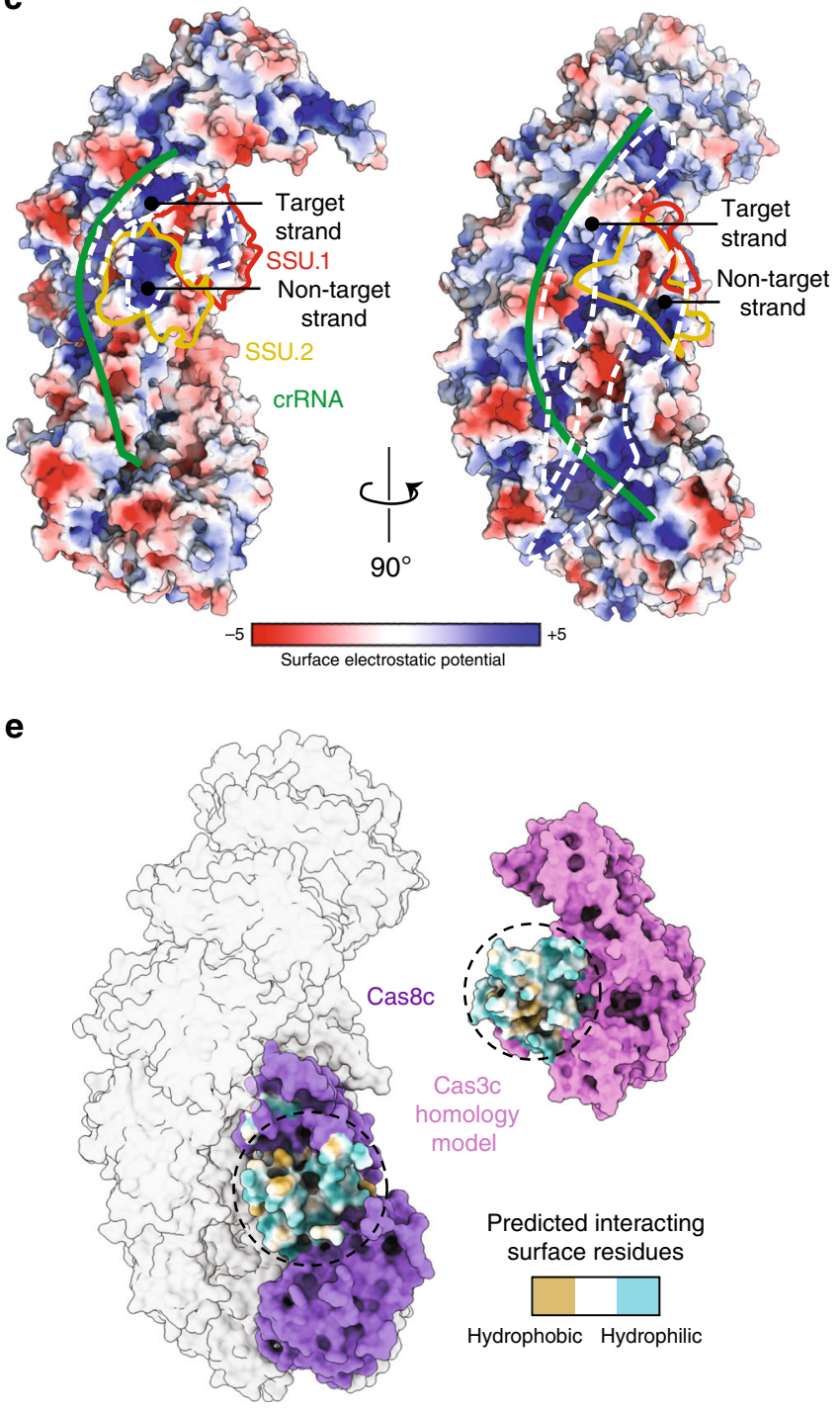

b

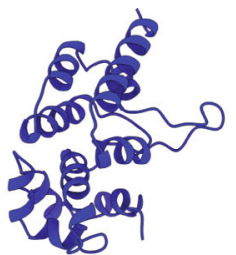

Type I-E

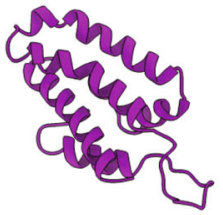

Type III-A

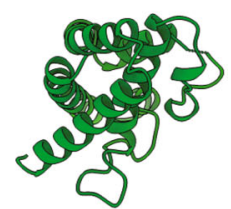

Type III-B

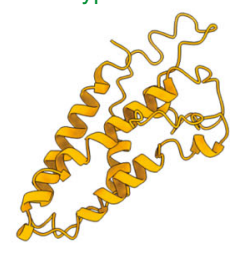

Type I-F C-terminus

\section{d}

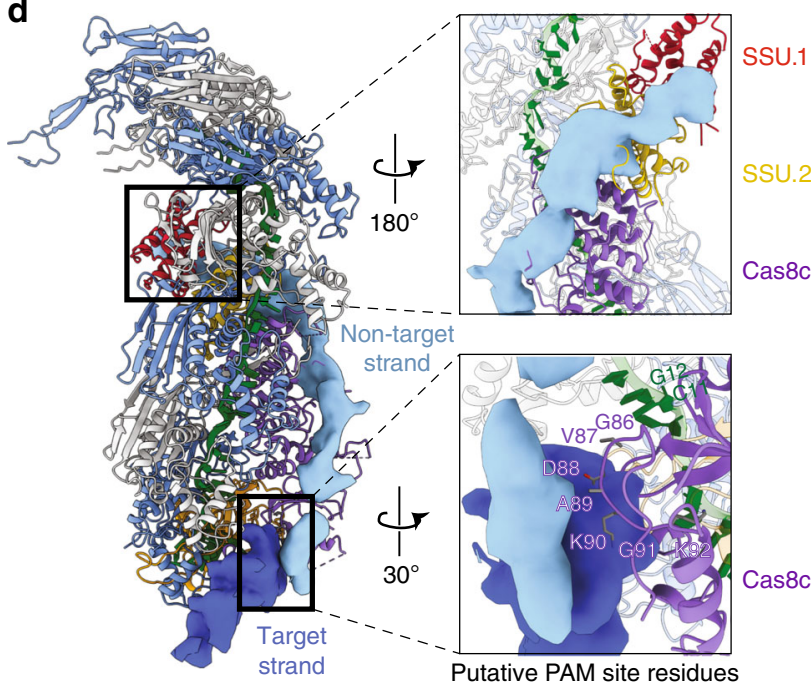

f

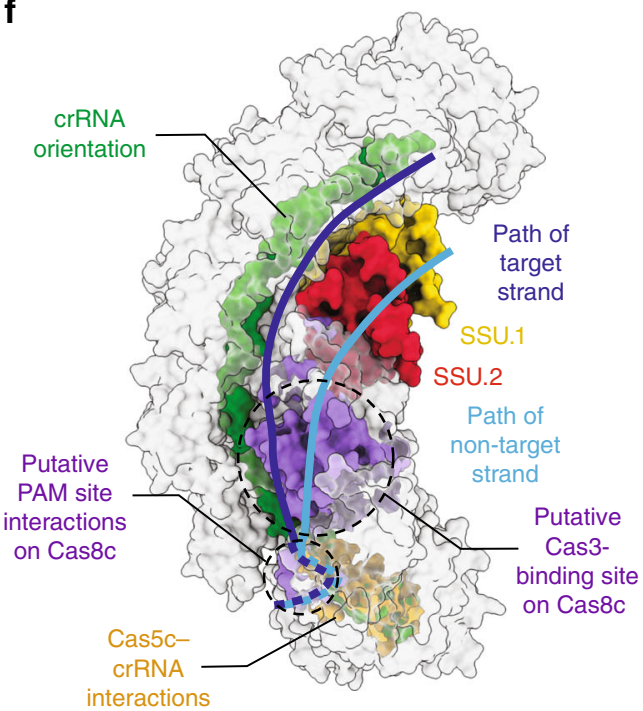

Fig. 2 Non-canonical small subunits (SSU) support R-loop formation. a Cas11 SSU are identical to the C terminus of Cas8c. b Canonical SSU found in type I-E, type III-A, type III-B, and the C-terminal domain of the type I-F large subunit aligned to the type I-C SSU. These SSUs are all helical bundles. c A channel of positively charged surface residues show Cas11c SSU likely accommodate the non-target strand during R-loop formation. $\mathbf{d}$ Putative Cas8c (purple) PAM site interacting residues (gray) determined by overlaying the dsDNA density (blue) from previous type I-C cryo-EM maps. e Cas8c (purple) and a homology model Cas3c (pink) show complementary surfaces for Cas3 docking. $\mathbf{f}$ Model of type I-C Cascade function. 
systems in bacteria which may serve as a blueprint for developing a minimal Cascade for genome editing ${ }^{24,25}$.

\section{Methods}

Protein purification. The D. vulgaris type I-C Cascade (addgene plasmid \#81185) and its crRNA (addgene plasmid \#81186) were co-expressed in NiCo21(DE3) E. coli cells. Cells were grown at $37^{\circ} \mathrm{C}$ to an $\mathrm{OD}_{600}$ of $0.6-0.8$ and induced by the addition of $0.5 \mathrm{mM}$ isopropyl- $\beta$-D-thiogalactopyranoside. After overnight growth at $18^{\circ} \mathrm{C}$, the cells were harvested and lysed by sonication in a buffer containing $50 \mathrm{mM}$ HEPES-NaOH ( $\mathrm{pH} 7.5$ ), $500 \mathrm{mM} \mathrm{KCl}, 10 \%$ (v/v) glycerol, $1 \mathrm{mM}$ tris(2carboxyethyl)phosphine (TCEP), 0.01\% Triton X-100, $0.5 \mathrm{mM}$ PMSF, and complete mini protease inhibitor tablets. The lysate was centrifuged at $27,000 \times g$ and incubated with Ni-NTA affinity resin overnight. The protein-bound resin was centrifuged and washed with buffer containing $50 \mathrm{mM}$ HEPES-NaOH $(\mathrm{pH} 7.5)$, $500 \mathrm{mM} \mathrm{KCl}, 20 \mathrm{mM}$ imidazole, $10 \%$ (v/v) glycerol, and $1 \mathrm{mM}$ TCEP. Protein was eluted with $50 \mathrm{mM}$ HEPES-NaOH (pH 7.5), $500 \mathrm{mM} \mathrm{KCl,} 300 \mathrm{mM}$ imidazole, $10 \%$ $(\mathrm{v} / \mathrm{v})$ glycerol, and $1 \mathrm{mM}$ TCEP. Approximately $1 \mathrm{mg}$ of TEV protease was added per $25 \mathrm{mg}$ of protein and the protein-TEV mixture was dialyzed at $4{ }^{\circ} \mathrm{C}$ overnight against size-exclusion buffer. The protein was then concentrated to approximately $10 \mathrm{mg} / \mathrm{mL}$ and run over a Superdex 200 Increase 10/300 GL size-exclusion column in a buffer containing $50 \mathrm{mM}$ HEPES-NaOH (pH 7.5), $500 \mathrm{mM} \mathrm{KCl,} \mathrm{5 \%} \mathrm{(v/v)}$ glycerol, and $1 \mathrm{mM}$ TCEP. The proteins were analyzed for purity by $10-20 \%$ SDSPage (Fig. S1) and then dialyzed overnight into the storage buffer containing $20 \mathrm{mM}$ HEPES-NaOH (pH 7.5), $100 \mathrm{mM} \mathrm{KCl,5 \%}$ (v/v) glycerol, and $1 \mathrm{mM} \mathrm{TCEP.}$ All proteins were finally concentrated, flash frozen in liquid nitrogen, and stored at $-80^{\circ} \mathrm{C}$. Source data is provided in the source data file.

Mass spectrometry. Prior to mass spectrometric analysis, the CRISPR complex solution buffer was exchanged to $100 \mathrm{mM}$ ammonium acetate using Micro Biospin P-6 gel columns (Bio-Rad Laboratories Inc., Hercules, CA). MS measurements were performed in positive mode using a Thermo Scientific Q Exactive Plus UHMR instrument (Bremen, Germany). Samples were loaded into gold/palladiumcoated borosilicate capillaries fabricated in-house. An electrospray voltage of $1.0 \mathrm{kV}$ was applied. The concentration of the CRISPR complex in solution was estimated as $\sim 6 \mu \mathrm{M}$. Trapping gas pressure was set to $10\left(\sim 1.0 \times 10^{-9}\right.$ mbar $)$ for high mass analysis and to $1-3\left(\sim 1.0 \times 10^{-10}-2.5 \times 10^{-10} \mathrm{mbar}\right)$ for low mass analysis. For the detection of the subunits, the in-source-trapping voltage (ranging from -100 to $-300 \mathrm{~V}$ ) was optimized for the release and transmission of the individual proteins as well as subcomplexes. In order to trap the macromolecular complexes, lower RF amplitudes of the bent flatapole and injection flatapole (range of $300 \mathrm{~V}$ instead of $900 \mathrm{~V})$ and IST voltages $(-120$ and $-300 \mathrm{~V})$ were used. MS1 and in-source trapping mass spectra were decharged and deisotoped using Xtract with a signalto-noise ratio of 2, fit factor of $44 \%$, and remainder of $25 \%$. Additionally, raw spectra were deconvoluted using UniDec ${ }^{26}$.

Cryo-EM sample preparation and data collection. Purified type I-C Cascade was diluted to a concentration of $0.3 \mathrm{mg} / \mathrm{mL}$ in a buffer containing $20 \mathrm{mM}$ HEPES-NaOH (pH 7.5), $100 \mathrm{mM} \mathrm{KCl}$, and $1 \mathrm{mM}$ TCEP. The CF-2/2 grids were first glow discharged for $60 \mathrm{~s}$ and then a layer graphene oxide was added 27,28 . Three microliters of protein were deposited on the grid and excess protein was blotted away after a $0.5 \mathrm{~s}$ incubation time for $4 \mathrm{~s}$ using filter paper at $4{ }^{\circ} \mathrm{C}$ in $100 \%$ humidity. The grid was then plunge frozen into liquid ethane using a Vitrobot Mark IV (Thermo Fisher). Frozen-hydrated samples of type I-C Cascade were directly visualized using a FEI Titan Krios microscope equipped with a Gatan K3 direct electron detector. Using the automated data-collection software LEGI$\mathrm{NON}^{29}$, we acquired $\sim 5400$ movies at a magnification of $\times 22,500$, corresponding to a calibrated pixel size of $1.047 \AA$ Aixel. A full description of the cryo-EM data collection parameters can be found in Table S2.

Cryo-EM data processing. Motion correction, CTF (contrast transfer function) estimation, and non-templated particle picking were performed in Warp ${ }^{30}$. Extracted particles were imported into CryoSPARC ${ }^{31}$ for $2 \mathrm{D}$ classification, $3 \mathrm{D}$ classification, and non-uniform 3D refinement. The final reconstruction was sharpened in CryoSPARC and subjected to density modification in PHENIX ${ }^{32,33}$. A final structure of type I-C Cascade at 3.13- $\AA$ resolution was determined using the 0.143 gold standard Fourier shell correlation-calculated from two independent half-sets-criterion. The model was built de novo in $\operatorname{Coot}^{34}$, and refined in PHENIX, ISOLDE ${ }^{35}$, and NAMDI$\mathrm{NATOR}^{36}$. The full cryo-EM data processing workflow is described in Fig. S2, and the model refinement statistics can be found in Table S2 and Fig. S3.

Reporting summary. Further information on research design is available in the Nature Research Reporting Summary linked to this article.

\section{Data availability}

The data that support the findings of this study are available from the corresponding author upon request. The cryo-EM structure of the type I-C minimal Cascade have been deposited into the Electron Microscopy Data Bank with accession number EMD-22876. The associated atomic models have been deposited into the Protein Data Bank with PDB code 7KHA. Source data are provided with this paper.

Received: 15 June 2020; Accepted: 30 October 2020; Published online: 23 November 2020

\section{References}

1. Wiedenheft, B., Sternberg, S. H. \& Doudna, J. A. RNA-guided genetic silencing systems in bacteria and archaea. Nature 482, 331-338 (2012).

2. Barrangou, R. et al. CRISPR provides acquired resistance against viruses in prokaryotes. Science 315, 1709-1712 (2007).

3. Makarova, K. S. et al. Evolutionary classification of CRISPR-Cas systems: a burst of class 2 and derived variants. Nat. Rev. Microbiol. 18, 67-83 (2020).

4. Makarova, K. S. et al. An updated evolutionary classification of CRISPR-Cas systems. Nat. Rev. Microbiol. Lond. 13, 722-736 (2015).

5. Nam, K. H. et al. Cas5d protein processes pre-crRNA and assembles into a cascade-like interference complex in subtype I-C/Dvulg CRISPR-Cas system. Structure 20, 1574-1584 (2012)

6. Hochstrasser, M. L., Taylor, D. W., Kornfeld, J. E., Nogales, E. \& Doudna, J. A DNA targeting by a minimal CRISPR RNA-guided cascade. Mol. Cell 63, 840-851 (2016)

7. McBride, T. M. et al. Diverse CRISPR-Cas complexes require independent translation of small and large subunits from a single gene. https://doi.org/ 10.1101/2020.04.18.045682 (2020).

8. Leney, A. C. \& Heck, A. J. R. Native mass spectrometry: what is in the name? J Am. Soc. Mass Spectrom. 28, 5-13 (2017).

9. Liko, I., Allison, T. M., Hopper, J. T. \& Robinson, C. V. Mass spectrometry guided structural biology. Curr. Opin. Struct. Biol. 40, 136-144 (2016).

10. Chorev, D. S., Ben-Nissan, G. \& Sharon, M. Exposing the subunit diversity and modularity of protein complexes by structural mass spectrometry approaches. Proteomics 15, 2777-2791 (2015).

11. Hernández, H. \& Robinson, C. V. Determining the stoichiometry and interactions of macromolecular assemblies from mass spectrometry. Nat. Protoc. 2, 715-726 (2007).

12. Duijn, E. et al. Native tandem and ion mobility mass spectrometry highlight structural and modular similarities in Clustered-Regularly-Interspaced ShotPalindromic-Repeats (CRISPR)-associated protein complexes from Escherichia coli and Pseudomonas aeruginosa. Mol. Cell. Proteomics 11, 1430-1441 (2012).

13. Jackson, R. N. et al. Structural biology. Crystal structure of the CRISPR RNAguided surveillance complex from Escherichia coli. Science 345, 1473-1479 (2014).

14. Chowdhury, S. et al. Structure reveals mechanisms of viral suppressors that intercept a CRISPR RNA-guided surveillance complex. Cell 169, 47-57.e11 (2017).

15. You, L. et al. Structure studies of the CRISPR-Csm complex reveal mechanism of co-transcriptional interference. Cell 176, 239-253.e16 (2019).

16. Osawa, T., Inanaga, H., Sato, C. \& Numata, T. Crystal structure of the CRISPR-Cas RNA silencing Cmr complex bound to a target analog. Mol. Cell 58, 418-430 (2015)

17. Xiao, Y. et al. Structure basis for directional R-loop formation and substrate handover mechanisms in type I CRISPR-Cas system. Cell 170, 48-60.e11 (2017)

18. Mojica, F. J. M., Díez-Villaseñor, C., García-Martínez, J. \& Almendros, C. Short motif sequences determine the targets of the prokaryotic CRISPR defence system. Microbiology 155, 733-740 (2009).

19. Hayes, R. P. et al. Structural basis for promiscuous PAM recognition in type IE cascade from E. coli. Nature 530, 499-503 (2016)

20. Hochstrasser, M. L. et al. CasA mediates Cas3-catalyzed target degradation during CRISPR RNA-guided interference. Proc. Natl Acad. Sci. USA 111, 6618-6623 (2014).

21. Xiao, Y., Luo, M., Dolan, A. E., Liao, M. \& Ke, A. Structure basis for RNAguided DNA degradation by Cascade and Cas3. Science 361, eaat0839 (2018).

22. Dillard, K. E. et al. Assembly and translocation of a CRISPR-Cas primed acquisition complex. Cell 175, 934-946.e15 (2018).

23. McCafferty, C. L., Marcotte, E. M. \& Taylor, D. W. Simplified geometric representations of protein structures identify complementary interaction interfaces. Proteins. In press (2020).

24. Morisaka, H. et al. CRISPR-Cas3 induces broad and unidirectional genome editing in human cells. Nat. Commun. 10, 5302 (2019).

25. Csörgő, B. et al. A compact Cascade-Cas3 system for targeted genome engineering. Nat. Methods 1-8. https://doi.org/10.1038/s41592-020-00980-w (2020).

26. Reid, D. J. et al. MetaUniDec: high-throughput deconvolution of native mass spectra. J. Am. Soc. Mass Spectrom. 30, 118-127 (2019).

27. Martin, T. G., Boland, A., Fitzpatrick, A. W. P. \& Scheres, S. H. W. Graphene oxide grid preparation. https://doi.org/10.6084/m9.figshare.3178669.v1 (2016). 
28. Palovcak, E. et al. A simple and robust procedure for preparing grapheneoxide cryo-EM grids. J. Struct. Biol. 204, 80-84 (2018).

29. Potter, C. S. et al. Leginon: a system for fully automated acquisition of 1000 electron micrographs a day. Ultramicroscopy 77, 153-161 (1999).

30. Tegunov, D. \& Cramer, P. Real-time cryo-electron microscopy data preprocessing with Warp. Nat. Methods 16, 1146-1152 (2019).

31. Punjani, A., Rubinstein, J. L., Fleet, D. J. \& Brubaker, M. A. cryoSPARC: algorithms for rapid unsupervised cryo-EM structure determination. Nat. Methods 14, 290-296 (2017).

32. Adams, P. D. et al. PHENIX: a comprehensive Python-based system for macromolecular structure solution. Acta Crystallogr. D Biol. Crystallogr. 66, 213-221 (2010).

33. Terwilliger, T. C. et al. Improvement of cryo-EM maps by density modification. Nat. Methods 17, 923-927 (2020).

34. Emsley, P. \& Cowtan, K. Coot: model-building tools for molecular graphics. Acta Crystallogr. D Biol. Crystallogr. 60, 2126-2132 (2004).

35. Croll, T. I. ISOLDE: a physically realistic environment for model building into low-resolution electron-density maps. Acta Crystallogr. Sect. Struct. Biol. 74, 519-530 (2018).

36. Kidmose, R. T. et al. Namdinator-automatic molecular dynamics flexible fitting of structural models into cryo-EM and crystallography experimental maps. IUCrJ 6, 526-531 (2019).

\section{Acknowledgements}

We thank Z. Zhou and C. McCafferty for help with sample freezing and protein interface predictions, respectively. Data were collected at the Sauer Structural Biology Laboratory at The University of Texas at Austin. This work was supported in part by Welch Foundation grants F-1155 (to J.S.B.) and F-1938 (to D.W.T.), Army Research Office Grant W911NF-15-1-0120 (to D.W.T.), the National Institute of General Medical Sciences (NIGMS) of the National Institutes of Health (NIH) (R01GM121714) (to J.S.B), and a Robert J. Kleberg, Jr. and Helen C. Kleberg Foundation Medical Research Award (to D.W.T.). D.W.T is a CPRIT Scholar supported by the Cancer Prevention and Research Institute of Texas (RR160088) and an Army Young Investigator supported by the Army Research Office (W911NF-19-1-0021).

\section{Author contributions}

R.E.O. purified and reconstituted the complex. R.E.O., D. W., and E.A.S. collected and processed the cryo-EM data. R.E.O. built and refined the model. I.C.S and J.S.B. conducted the native mass spectrometry experiments and provided analysis of the data R.E.O., J.P.K.B., and D.W.T. interpreted the cryo-EM results. R.E.O. and D.W.T. wrote the manuscript with input from all authors. D.W.T. conceived the experiments, supervised the research, and secured funding for the project.

\section{Competing interests}

The authors declare no competing interests.

\section{Additional information}

Supplementary information is available for this paper at https://doi.org/10.1038/s41467020-19785-8.

Correspondence and requests for materials should be addressed to D.W.T.

Peer review information Nature Communications thanks Kallol Gupta and other, anonymous reviewers for their contributions to the peer review of this work. Peer review reports are available.

Reprints and permission information is available at http://www.nature.com/reprints

Publisher's note Springer Nature remains neutral with regard to jurisdictional claims in published maps and institutional affiliations.

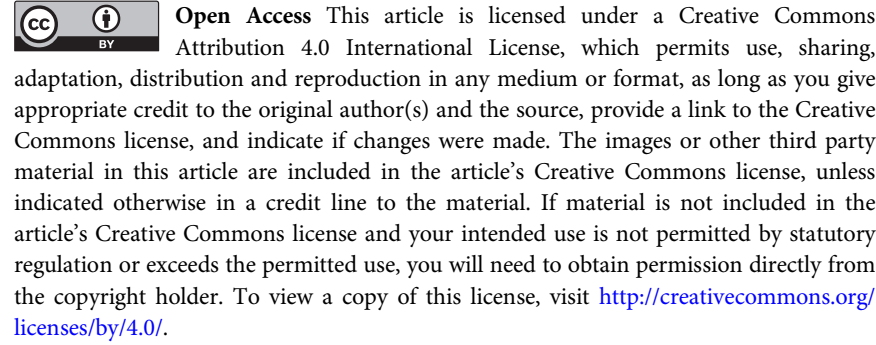

(C) The Author(s) 2020 